\title{
A Brief Review: Antibacterial Activity of Quinone Derivatives
}

\author{
Patitapaban Mohanty Subhasmita Sahoo ', Sunita Behera ', Rubi Behura ', Anantjyoti Acharya 1, \\ Debajyoti Biswal ${ }^{1}$, Sameer Kumar Suna ${ }^{1}$, Rojalin Sahoo ${ }^{1}$, Ram Chandra Soren ${ }^{1}$, \\ Bigyan Ranjan Jali ${ }^{1, *}$ (iD \\ 1 Department of Chemistry, Veer Surendra Sai University of Technology, Burla, Sambalpur-768018, Odisha, India \\ * Correspondence: bigyan.Jali7@ gmail.com (B.R.J.);
}

Scopus Author ID 36503969200

Received: 16.06.2021; Revised: 25.07.2021; Accepted: 29.07.2021; Published: 8.08.2021

\begin{abstract}
Quinones and their derivatives play an important role in pharmaceutical, medicinal, and environmental applications. The biological activities of quinone derivatives against various grampositive and negative bacteria and pathogens show strategies for discovering new drug efficiency and various physio-chemical properties. This review discusses the antibacterial activities of various notable quinones and their derivatives against various bacteria and pathogens. A wide literature survey is done on the database PubMed, Scopus, and Google scholars by using code word antibacterial activity of quinone derivatives and the role of various quinone derivatives to discover new potent pharmaceutical applications. Herein, we wish to discuss various quinone derivatives used as potent drug molecules for antibacterial activity against various gram-positive and negative bacteria and pathogens. Proper health care systems are essential for newly develop methodologies to fight against unexpected bacteria and pathogens. The antibacterial activities of various quinone derivatives deliver notable supervision for additional design and synthesis of quinone derivatives and examine the pharmaceutical, industrial and medicinal applications.
\end{abstract}

Keywords: quinone; naphthoquinone; anthraquinone, anti-bacteria; activity; drug; bacteria.

(c) 2021 by the authors. This article is an open-access article distributed under the terms and conditions of the Creative Commons Attribution (CC BY) license (https://creativecommons.org/licenses/by/4.0/).

\section{Introduction}

Due to their unique physical and chemical properties, quinones and their derivatives play a vital role in chemical, environmental, and pharmaceutical applications [1-7]. Quinones and their derivatives are biologically active compounds and spectacle a significant role in electron-transfer and photochemical processes [8-12]. They are widely present in nature [13]. They are mainly obtained from plants, animals, bacteria, and fungi in the direct way or inindirect way $[14,15]$. Quinone derivatives ascribe an important role in various fields such as chemosensor, MOF, catalyst, ROS, polymorphs, dyes, batteries, energy storage, electron transfer, etc. [16-25]. Apart from that, the natural or synthetic quinone derivatives are prominent members of viable or potential drug molecules in the medicinal and pharmaceutical industry [27-29]. Due to their sole pharmaceutical applications are widely used as anticancer, anti-oxidant, anti-malaria, antimicrobial, anti-inflammatory agents [30,31]. Quinone derivatives arouse many clinical and pharmaceutical applications such as phylloquinone, $\beta$ lapachone, lapachol, lawsone, plumbagin, juglomycin A, menadione, etc. (Figure 1). It is well documented that quinone moiety in the vitamin $\mathrm{K}_{1}$ and $\mathrm{K}_{2}$ is chiefly responsible for various biological activities [32-34]. Due to their assorted utilities and clinical applications, quinone 
derivatives are widely used to develop novel antibacterial drug molecules. Herein reported the systematic evaluation of various quinone derivatives and their antibacterial activity against various bacteria and pathogens using different methods.<smiles>CC(=CCC1=C(C)C(=O)c2ccccc2C1=O)CCCC(C)(C)C</smiles>

Vitamin K (i)<smiles>CC(C)=CCC1=C(O)C(=O)c2ccccc2C1=O</smiles>

Lapachol (ii)<smiles>C[C@H]1O[C@@H](O[C@H]2C[C@H](O)[C@@H](O)[C@H](N)C2)Cc2c(O)c3c(c(O)c21)C(=O)c1c(O)cccc1C3=O</smiles><smiles>O=C1C(Cl)=C(Nc2ccc(O)cc2)C(=O)c2ccccc21</smiles>

(iv)<smiles>Cc1ccc(NC2=C(Br)C(=O)c3ccccc3C2=O)nc1</smiles>

(v)<smiles>O=C1C(Cl)=C(N2CCCC2)C(=O)c2ccccc21</smiles>

(vi)<smiles>COCNC1=CC(=O)c2ccccc2C1=O</smiles>

(vii)<smiles>COc1cc(C2=CC(=O)c3ccccc3C2=O)cc(N(C)C)c1</smiles>

(viii)<smiles>CCCCC(C1=C(O)C(=O)c2ccccc2C1=O)C(Br)CC</smiles>

(ix)<smiles>O=C1C(Cl)=C(NCCO)C(=O)c2ccccc21</smiles>

(x)<smiles>NS(=O)(=O)c1cccc(NC2=CC(=O)c3ccccc3C2=O)c1</smiles>

(xi)

Figure 1. A few examples of natural and synthetic quinone derivatives as antibacterial agents.

\section{Result and Discussions}

In the field of the clinical world, consideration of retardation of pathogenic bacteria towards the available antibiotic is becoming a major worldwide problem as many bacterial pathogens have already established resistance against them [35-37]. To achieve a new effective scaffold for an efficient fight against bacteria. The research on the development of a new antimicrobial agent has mainly focused on two aspects like (i) increasing potent bacterial antigens and (ii) appearance of new bacteria pathogen. The main aspect of synthesizing effective drugs is their structural characteristic and the rate of activity. In this regard, quinones and their derivatives play an essential role in developing a novel-anti-bacterial drug against various bacteria and pathogens. It is found that quinone derivatives displayed powerful antibacterial activity, and also these derivatives were used for various pharmaceutical and clinical applications [38-40]. In this regard, Gawali et al. reported two novel 1,4naphthoquinone derivatives (1-2) and examined their antibacterial activity against various pathogens. From the experimental data, it is observed that probe $\mathbf{1}$ exhibited potent antibacterial activity against $S$. aureus NCIM 2079 and $P$. aeruginosa MTCC 2297. On the other hand, probe 2 showed better antibacterial activity against all pathogens with MIC values in the range of $32-512 \mu \mathrm{g} / \mathrm{ml}$. Due to their differential structural activity, both derivatives ascribed better activity compared to the reference antibiotics [41]. Antibacterial activities of a series of novel 1,4-benzoquinones derivatives (3-4a-h) were demonstrated against various gram-positive and negative bacteria using the micro broth dilutions method. The authors 
suggested that 3a-b displayed better antibacterial activity against $S$. epidermidis and Amikacin with MIC values of $4.88 \mu \mathrm{g} / \mathrm{mL}$ and $78.12 \mu \mathrm{g} / \mathrm{mL}$ as compared to Cefuroxime as a reference drug molecule. On the other hand, the other derivatives remain silent towards the various bacteria [42]. Similarly, Kijja et al. reported a series of 1,3-Dioxepine and spiropyran derivatives (5-7) and determined the antibacterial activity against various pathogens. The experimental data found that 5-7 show better antibacterial activity against gram-positive bacteria, including Staphylococcus aureus ATCC 29213, Enterococcus faecalis ATCC 29212, and environmental isolates $S$. aureus with reference strains [43]. A series of novel 1,4naphthoquinone derivatives (8-11) was designed and evaluated against raw cell line's antibacterial activity. From the above data, it is found that $\mathbf{8}$ and $\mathbf{9}$ showed the highest antibacterial activities against raw cell lines with $\mathrm{IC}_{50}$ values of 483.5-2044. $8 \mathrm{M}$ [44]. Antibacterial activities of ortho-quinone derivative (12) against some medicinal plants were demonstrated. The authors suggested that $\mathbf{1 2}$ ascribe potent antibacterial activity against grampositive bacteria, including Staphylococcus aureus, S. epidermidis, Enterococcus faecalis, and Micrococcus luteus [45]. A series of novel diaziridinyl quinone isoxazole hybrids (13a-j) was reported by Yennam et al. All the synthesized derivatives were used to detect the antibacterial activity against A549 and PC3 Cell lines. It was observed that 13h show the highest antibacterial activity towards Staphylococcus aureus MTCC 96, S. aureus MLS-16 MTCC 2940, Bacillus subtilis MTCC 121, and Klebsiella planticola MTCC 530 [46]. 1,4Napthoquinone derivatives (14a-e) showed antibacterial activity against multi-resistant bacterial strains. The authors found that $\mathbf{1 4 b}$-d shows the highest antibacterial activity against gram-positive bacteria (Staphylococcus aureus) and negative bacteria (Pseudomonas aeruginosa, Escherichia coli I, Escherichia coli 12 and Klebsiella pneumonia) with MIC value $0.30 \mathrm{mg} / \mathrm{mL}$ and $1.05 \mathrm{mg} / \mathrm{mL}$ [47].

Antibacterial activities of a series of novel quinone derivatives (15a-h) were demonstrated against several bacterial strains and fungi. The authors suggested that $\mathbf{1 5 a}$ shows the highest antibacterial activity against gram-positive and negative bacteria towards $S$.aureus $(\mathrm{MIC}=39.06 \mu \mathrm{g} / \mathrm{mL})$ [48]. A series of NH or S-substituted 1,4-quinones (16-23) was demonstrated and estimated their antibacterial activity against gram-positive and gramnegative bacteria. The experimental data shows that 22-23 show the potent antibacterial activity against E.Coli, Staphylococcus aureus, Microsporum canis, and Trichophyton mentagrophytes [49].<smiles>O=C1C(Cl)=C(Nc2cccc(O)c2)C(=O)c2ccccc21</smiles><smiles>O=C1C(Cl)=C(Nc2ccc(O)cc2)C(=O)c2ccccc21</smiles>

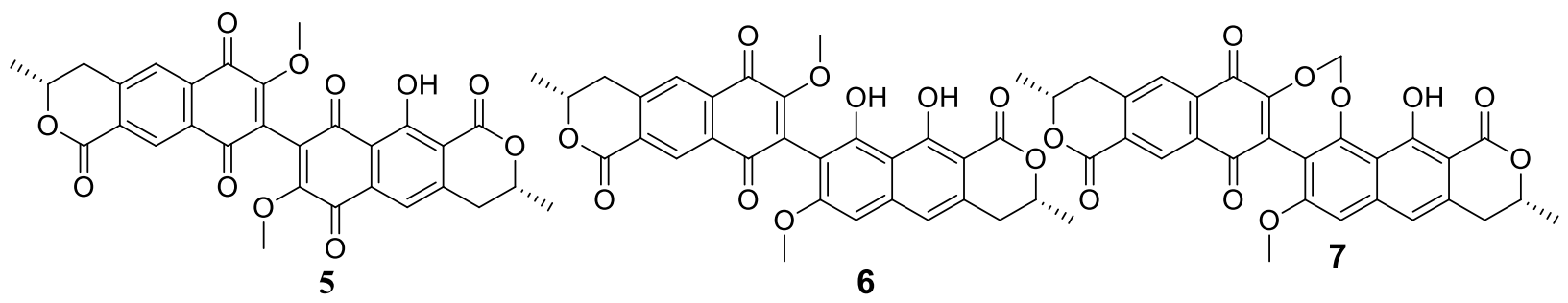


<smiles>[R]C1=C(Cl)C(=O)C(C)=C(C)C1=O</smiles>

3a-h<smiles>[R]C1=CC(=O)C(C)=C(C)C1=O</smiles>

4a-h<smiles>[R][14CH2][14CH2]N1CCN(C(C)C)CC1</smiles>

3d/4d:<smiles>Cc1cccc(N2CCN(C)CC2)c1</smiles>

3e/4e:<smiles>Cc1ccc(N2CCN(C)CC2)cc1</smiles><smiles>COc1ccc(N2CCN(C)CC2)cc1</smiles>

3h/4h:<smiles>COc1ccccc1N1CCN(C)CC1</smiles>

3g/4g:<smiles>COc1cccc(N2CCN(C)CC2)c1</smiles><smiles>O=C1C(O)=C(Nc2ccccc2)C(=O)c2ccccc21</smiles>

8<smiles>O=C1C(O)=C(Nc2ccccc2Cl)C(=O)c2ccccc21</smiles>

9<smiles>COc1ccc(NC2=C(O)C(=O)c3ccccc3C2=O)cc1</smiles>

10<smiles>Cc1cccc(C)c1NC1=C(O)C(=O)c2ccccc2C1=O</smiles>

11<smiles>CC1=C(C)C2CC(=O)C3=C(C(=O)C(=O)[C@H](O)[C@H]3C(C)C)[C@]2(C)CC1</smiles>

12<smiles>[R]N([R])C(=O)c1ccc(-c2cc(C3=C(N4CC4)C(=O)C=C(N4CC4)C3=O)no2)s1</smiles>

13a-j

13a, $\mathbf{R}_{1}=\mathbf{R}_{2}=\mathrm{Me} ; \mathbf{1 3 b}, \mathbf{R}_{1}=\mathbf{R}_{\mathbf{2}}=\mathrm{Et} ; \mathbf{1 3 c}, \mathbf{R}_{\mathbf{1}}=\mathrm{Me}, \mathbf{R}_{\mathbf{2}}=$ Propyl;

13d, $R_{1}=H, R_{2}=$ Methylcuclopropane; 13e, $R_{1}=R_{2}=$ Pyrrolidinyl;

13f, $\mathbf{R}_{\mathbf{1}}=\mathbf{R}_{\mathbf{2}}=$ Piperidinyl; 13g, $\mathbf{R}_{\mathbf{1}}=\mathbf{R}_{\mathbf{2}}=4$ (trifluoromethyl)piperidinyl;

13h, $\mathbf{R}_{1}=\mathbf{R}_{2}=$ Morpholinyl; 13i, $\mathbf{R}_{1}=\mathrm{Me}, \mathbf{R}_{2}=$ Benzyl; 13j, $\mathbf{R}_{1}=\mathrm{Me}, \mathbf{R}_{2}=4-$ fluoro benzyl<smiles>[R]C#CC1=C(Cl)C(=O)c2ccccc2C1=O</smiles>

14a-e

$14 \mathbf{a}=\equiv 14 \mathbf{b}=\equiv \underbrace{\mathrm{OH}} 14 \mathbf{c}=\equiv \underbrace{\mathrm{OH}} 14 \mathrm{~d}=\equiv \mathrm{C}_{4} \mathrm{H}_{10} \quad 14 \mathrm{e}=\equiv \mathrm{C}_{6} \mathrm{H}_{13}$

Figure 2. Various quinone derivatives used for antibacterial studies. 
A series of novel 1,4-Napthaquinone derivatives (24-25) were reported by Pisoschi et $a l$. The antibacterial activity of these derivatives was examined against various bacteria. It is observed that all compounds display better antibacterial activity against gram-positive bacteria by diffusion method [50]. Cunha et al. reported a series of novel derivatives (26a-d) and evaluated the antibacterial properties against some pathogenic strains. The authors found that 26d shows potent antibacterial activity against gram-negative bacteria towards $E$. coli and $P$. aeruginosa. The MIC value was calculated and found to be $1-2 \mu \mathrm{g} \mathrm{mL}^{-1}$ [51]. A series of novel 2,3-Disubstituted-1,4-naphthoquinone derivatives (27-30) was demonstrated by Yildirim et al. and estimated they are in vitro antibacterial activity against several bacterial strains and yeast. The experimental analysis revealed that the derivatives $27 \mathrm{~b}$ and $28 \mathrm{~b}$ show excellent antibacterial activity against various human-originated pathogens with MIC values 4.88 and $2.44 \mu \mathrm{g} / \mathrm{mL}$ compared to reference drug molecules such as Cefuroxime [52]. Antibacterial activities of a few naphthoquinones derivatives were demonstrated against various pathogens and bacteria. The antibacterial data found that the derivatives (31-33) show excellent activity against various pathogens [53]. Two novels 1,2-naphthoquinone derivatives (34-35), were reported and synthesized by Duan et al. The antibacterial activity was demonstrated against various gram-positive and negative bacteria. Both derivatives display excellent antibacterial activity against gram-positive bacteria [54]. Antibacterial activities of a series of various quinone derivatives were demonstrated against various bacterial. The authors found that 3'methyl-6-(methylthio)-[1,1'-biphenyl]-2,5-dione (36) ascribe potent antibacterial activity [55]. A series of novel quinone derivatives (37a-l) were demonstrated antibacterial activity against various gram-positive and negative bacteria. From the antibacterial analysis, it is observed that ten derivatives envisage the potent antibacterial activity against various gram-negative bacteria with MIC values 4.00 to $64 \mu \mathrm{g} / \mathrm{mL}$ [56]. Antibacterial activities of a series of novel naphthoquinone derivatives (38-45) were demonstrated against various gram-positive and negative bacteria. The antibacterial studied shows that the derivative (38) displays strong antibacterial activity, and MIC value is calculated and found to be 200 to $400 \mu \mathrm{g} / \mathrm{mL}$.

On the other hand, the authors suggested that $\mathbf{4 1}$ envisaged moderate antibacterial activity. The MIC value is found to be $25 \mu \mathrm{g} / \mathrm{mL}$. The other compounds remain silent towards bacteria [57].<smiles>[R]c1nc2c(s1)C(=O)c1ccccc1C2=O</smiles>

15a-h

$15 \mathrm{a}=$

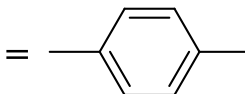

$15 b=$<smiles>Cc1ccc(F)cc1</smiles>

$15 \mathrm{c}=$<smiles>Cc1ccc(Cl)cc1</smiles>

$15 d=$<smiles>Cc1ccc(Br)cc1</smiles><smiles>OCc1ccccc1C#[Al]</smiles><smiles>CCC=[As]</smiles>

$15 h=$<smiles>CCCc1ccccc1</smiles> 
<smiles>CCC(C)N(C)CCCNC1=C(Br)C(=O)c2ccccc2C1=O</smiles>

16

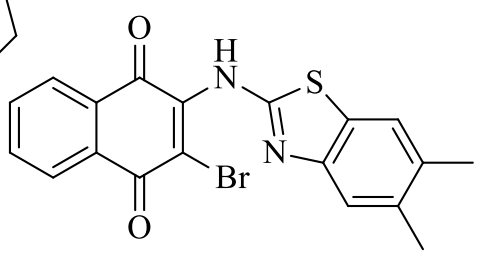

17<smiles>O=C1C(Br)=C(Nc2ccc(F)cc2)C(=O)c2ccccc21</smiles>

18

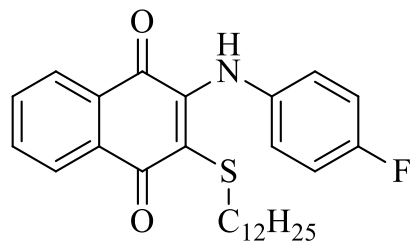

19<smiles>Cc1cc(C)cc(SC2=CC(=O)C=CC2=O)c1</smiles><smiles>[R]NC1=C(Cl)C(=O)c2ccccc2C1=O</smiles><smiles>[Z16][Y16]=Cc1cccc(O)c1</smiles>

$\mathbf{2 4} \mathbf{c}={ }_{\mathrm{OH}}^{\mathrm{COOH}} \mathbf{2 4 d}=-\mathrm{CHN}$<smiles>[R20]C=[R10]</smiles><smiles>[R]NC1=C(SC(=N)N)C(=O)c2ccccc2C1=O</smiles><smiles>Cc1nccs1</smiles><smiles>[C]#Cc1ccc(C(=O)O)c(C)c1</smiles><smiles>Nc1ccc(NC=[Zn])c(N)c1</smiles>

$\mathbf{2 6} \mathbf{a}=\mathrm{R} 1=\mathrm{R} 2=\mathrm{R} 3=\mathrm{H} \mathbf{2 6} \mathbf{b}=\mathrm{R} 1=\mathrm{OMe}, \mathrm{R} 2=\mathrm{R} 3=\mathrm{H} \mathbf{2 6} \mathbf{c}=\mathrm{R} 1=\mathrm{R} 2=\mathrm{H}, \mathrm{R} 3=\mathrm{OMe} \mathbf{2 6} \mathbf{d}=\mathrm{R} 1=\mathrm{R} 2=\mathrm{H}, \mathrm{R} 3=\mathrm{Cl}$ 


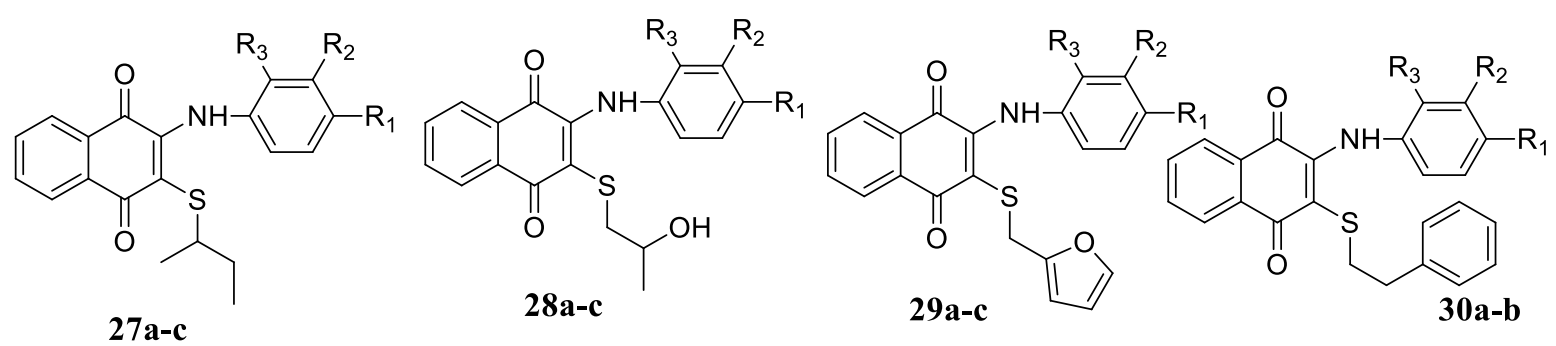

27a, R1 $=\mathrm{CF}_{3}, \mathrm{R} 2=\mathrm{R3}=\mathrm{H} 28 \mathrm{a}, \mathrm{R} 1=\mathrm{CF}_{3}, \mathrm{R} 2=\mathrm{R} 3=\mathrm{H} 29 \mathrm{a}, \mathrm{R} 1=\mathrm{CF}_{3}, \mathrm{R} 2=\mathrm{R} 3=\mathrm{H}$

$27 \mathrm{~b}, \mathrm{R2}=\mathrm{CF}_{3}, \mathrm{R} 1=\mathrm{R3}=\mathrm{H} 28 \mathrm{~b}, \mathrm{R2}=\mathrm{CF}_{3}, \mathrm{R} 1=\mathrm{R} 3=\mathrm{H} 29 \mathrm{~b}, \mathrm{R} 2=\mathrm{CF}_{3}, \mathrm{R} 1=\mathrm{R} 3=\mathrm{H}$

$27 \mathrm{c}, \mathrm{R3}=\mathrm{CF}_{3}, \mathrm{R} 2=\mathrm{R} 1=\mathrm{H} 2 \mathrm{28c}, \mathrm{R3}=\mathrm{CF}_{3}, \mathrm{R} 2=\mathrm{R} 1=\mathrm{H} 2 \mathrm{cc}, \mathrm{R3}=\mathrm{CF}_{3}, \mathrm{R} 2=\mathrm{R} 1=\mathrm{H}$

30a, R1 $=\mathrm{CF}_{3}, \mathrm{R2}=\mathrm{R3}=\mathrm{H}$

30b, R2 $=\mathrm{CF}_{3}, \mathrm{R} 1=\mathrm{R} 3=\mathrm{H}$<smiles>CCCOC(=O)CCSC1=C(SCCC(=O)OCCC)C(=O)c2ccccc2C1=O</smiles>

31<smiles>O=C1C(Cl)=C(SCCCO)C(=O)c2ccccc21</smiles>

32<smiles>CCOC1=C(SCCC(=O)OC)C(=O)c2ccccc2C1=O</smiles>

33<smiles>Cc1c(CN2CCCC2)oc2c1C(=O)C(=O)c1c-2ccc2c1CCCC2(C)C</smiles><smiles>Cc1c(CN2CCCC2)oc2c1C(=O)C(=O)c1c-2ccc2c(C)cccc12</smiles><smiles>CSC1=C(c2cccc(C)c2)C(=O)C=CC1=O</smiles><smiles>[R]SC(C1=C(O)C(=O)c2ccccc2C1=O)c1ccc(OC)cc1</smiles>

37a-I

$\mathrm{a}, \mathrm{R}=\mathrm{C}_{6} \mathrm{H}_{5} ; \mathrm{b}, \mathrm{R}=2-\mathrm{CH}_{3} \mathrm{C}_{6} \mathrm{H}_{4} ; \mathrm{c}, \mathrm{R}=3-\mathrm{CH}_{3} \mathrm{C}_{6} \mathrm{H}_{4} ; \mathrm{d}, \mathrm{R}=4-\mathrm{CH}_{3} \mathrm{C}_{6} \mathrm{H}_{4} ; \mathrm{e}, \mathrm{R}=4-\mathrm{ClC}_{6} \mathrm{H}_{4} ; \mathrm{f}, \mathrm{R}=4-$ $\mathrm{FC}_{6} \mathrm{H}_{4} ; \mathrm{g}, \mathrm{R}=4-\mathrm{NO}_{2} \mathrm{C}_{6} \mathrm{H}_{4} ; \mathrm{h}, \mathrm{R}=$ Propyl; i, R= naphthyl; j, R=4-CH $\mathrm{CC}_{6} \mathrm{H}_{4} ; \mathrm{k}, \mathrm{R}=4-\mathrm{CH}_{3} \mathrm{SC}_{6} \mathrm{H}_{4}$; l, $\mathrm{R}=4-\mathrm{OHC}_{6} \mathrm{H}_{4}$ 
<smiles>COC1=C(O)C(=O)c2c(O)c3c(c(OC)c2C1=O)C=C(C)OC3C</smiles><smiles>COc1ccc2c(c1)C(=O)c1ccc(OC)cc1C2=O</smiles><smiles>Cc1ccc2c(c1)C(=O)c1ccc(O)cc1C2=O</smiles><smiles>CCCC1OC(C)=Cc2cc3c(c(O)c21)C(=O)C=CC3=O</smiles>

42<smiles>CCCC1OC(C)=Cc2cc3c(c(O)c21)C(=O)C(C1OC[C@H](O)[C@H](CO)O1)C=C3C</smiles>

43<smiles>COC1=C(C)C(=O)c2c(cc3c(c2O)C(C)OC(C)C3)C1=O</smiles>

44<smiles>COC1=CC(=O)c2c(cc3c(c2O)C(C)OC(C)C3)C1=O</smiles>

45

Figure 3: Various quinone derivatives used for the antibacterial study.

\section{Conclusions and perspectives}

In this review, the antibacterial activities of various novel quinone derivatives such as naphthoquinones, benzoquinones, anthraquinones are designated. The antibacterial activity of quinone derivatives was enhanced due to the polarity of the substituents: the stronger polarity, the excellent antibacterial activity against various gram-positive and negative bacteria and pathogens. Various novel quinones have excellent bio-medical applications and wide-ranging arcade diagnoses. It is well known that investigation of various quinones and their derivatives possess significant biological activities, so it is of great interest to design and synthesize numerous novel quinones and their derivatives and examine their biological applications as a source of inspiration for upcoming therapeutic enlargement. This summary and review of antibacterial activities of various quinone derivatives deliver notable supervision for additional design and synthesis of quinone derivatives and their related drugs.

\section{Funding}

Dr. Jali acknowledges a fund from DST, India, by EMEQ project (No.-EEQ/2019/000091). The authors also thank the Department of Chemistry, VSSUT, Burla, for providing the research facility.

\section{Acknowledgments}

The authors thank the Department of Chemistry, VSSUT Burla, Sambalpur, Odisha, India

\section{Conflict of interest}

The authors have declared that no competing interests exist.

\section{References}

1. Jung, H. A.; Ali, M. Y.; Jung, H. J.; Jeong, H. O.; Chung, H. Y.; Choi, J. S. Inhibitory activities of major anthraquinones and other constituents from Cassia obtusifolia against beta-secretase and cholinesterases. $J$. Ethnopharmacol. 2016, 191, 152-160, https://doi.org/10.1016/j.jep.2016.06.037.

2. Jiang, J. G.; Huang, X. J.; Chen, J.; Lin,Q. S. Comparison of the sedative and hypnotic effects of flavonoids, saponins and polysaccharides extracted from Semen Ziziphus jujube. Nat. Prod. Res. 2007, 21, 310-320, https://doi.org/10.1080/14786410701192827. 
3. Liu, L. Q.; Tan, X. Y.; Zhao, C. X.; Wu, H. J.; Liu, P. Impact of compatibility ratios of rhubarb-gardenia hrbal pair and extraction techniques on anthraquinone dissolution. Chin. Tradit. Herb. Drugs 2017, 48, 283287, http://en.cnki.com.cn/Article_en/CJFDTotal-ZCYO201702016.htm.

4. Jali, B. R. A Mini-Review: Quinones and their Derivatives for Selective and Specific Detection of Specific Cations. Biointerface Res. Appl. Chem. 2021, 11, 11679-11699, https://doi.org/10.33263/BRIAC114.1167911699.

5. Thomson, R. H. Distribution of naturally occurring quinones. Pharm. Weekbl., Sci. Ed., 1991, 13, 70-73, https://doi.org/10.1007/BF01974983.

6. Vazquez, M. F. B.; Comini, L. R.; Martini, R. E.; Montoya, S. C. N.; Bottini, S.; Cabrera, J. L. Comparisons between conventional, ultrasound-assisted and microwave-assisted methods for extraction of anthraquinones from Heterophyllaea pustulata Hook f. (Rubiaceae), Ultrason. Sonochem. 2014, 21, 478-484, https://doi.org/10.1016/j.ultsonch.2013.08.023.

7. Balachandran, C., Al-Dhabi, N.A., Duraipandiyan, V. et al. Bluemomycin, a new naphthoquinone derivative from Streptomyces sp. with antimicrobial and cytotoxic properties. Biotechnol Lett 2021, 43, 1005-1018, https://doi.org/10.1007/s10529-021-03089-y.

8. Jali, B. R.; Barick, A. K.; Mohapatra, P.; Sahoo, S. K. A comprehensive review on quinones based fluoride selective colorimetric and fluorescence chemosensors. J. Fluor. Chem. 2021, 244, 109744, https://doi.org/10.1016/j.jfluchem.2021.109744.

9. Jali, B. R.; Kuang, Y.; Nemati, N.; Baruah, J. B., Selective binding of naphthoquinone derivatives to serum albumin proteins and their effects on cytotoxicity. Chem.-Biol. Interact 2014, 214, 10-17, https://doi.org/10.1016/j.cbi.2014.01.014.

10. Li, Y.; Guo, F.; Guan, Y.; Chen, T.; MA, K.; Zhang, L.; Wang, Z.; Su, Q.; Feng, L.; Liu, Y.; Zhou, Y. Novel Anthraquinone Compounds Inhibit Colon Cancer Cell Proliferation via the Reactive Oxygen Species/JNK Pathway. Molecules 2020, 25, 1672, https://doi.org/10.3390/molecules25071672.

11. Feng, S.; Wang, W. Bioactivities and Structure-Activity Relationships of Natural Tetrahydroanthraquinone Compounds: A Review. Front. Pharmacol. 2020, 11, 1-10, https://doi.org/10.3389/fphar.2020.00799.

12. Kacmaz, A.; Deniz, N. G.; Aydinli, S. G.; Sayil, C.; Onay-Ucar, E.; Mertoglu, E.; Arda, N. Synthesis and antiproliferative evaluation of some 1,4-naphthoquinone derivatives against human cervical cancer cells. Open Chem. 2019, 17, 337-345, https://10.1515/chem-2019-0030.

13. Pereyra,C. E.; Dantas, R. F.; Ferreira, S. B.; Gomes, L. P.; Silva-Jr, F. P. The diverse mechanisms and anticancer potential of naphthoquinones. Cancer Cell Int 2019, 19, 207, https://doi.org/10.1186/s12935-0190925-8.

14. Campora, M.; Francesconi, V.; Schenone, S.; Tasso, B.; Tonelli, M. Journey on Naphthoquinone and Anthraquinone Derivatives: New Insights in Alzheimer's Disease.

Pharmaceuticals 2021, 14, 33, https://doi.org/10.3390/ph14010033.

15. Ma, Q.; Wei, R.; Sang, Z. Structural Characterization and Hepatoprotective Activity of Naphthoquinone From Cucumis bisexualis. Nat. Prod. Commun. 2020, 15, 1-6, https://doi.org/10.1177/1934578X20902898.

16. Shi, Y.; Sun, P.; Yang, J.; Xu, Y. Benzoquinone- and Naphthoquinone-Bearing Polymers Synthesized by Ring-Opening Metathesis Polymerization as Cathode Materials for Lithium-Ion Batteries. ChemSusChem 2020, 13, 334-340, https://doi.org/10.1002/cssc.201902966.

17. Huang, R.; Jing, X.; Huang, X.; Pan, Y.; Fang, Y.; Liang, G.; Liao, Z.; Wang, H.; Chen, Z.; Zhang, Y. Bifunctional Naphthoquinone Aromatic Amide-Oxime Derivatives Exert Combined Immunotherapeutic and Antitumor Effects through Simultaneous Targeting of Indoleamine-2,3-dioxygenase and Signal Transducer and Activator of Transcription 3. J. Med. Chem. 2020, 63, 1544-1563, https://doi.org/10.1021/acs.jmedchem.9b01386.

18. Oliveira, K. M.; Peterson, E. J.; Carroccia, M. C.; Cominetti, M. R.; Deflon, V. M.; Farrell, N. P.; Batista, A. A.; Correa, R. S. Ru(ii)-Naphthoquinone complexes with high selectivity for triple-negative breast cancer. Dalton Trans. 2020, 49, 16193-16203, https://doi.org/10.1039/D0DT01091J.

19. Zhao, H.; Yang, A.; Liu, J.; Bao, S.; Peng, R.; Hu, Y.; Yuan, T.; Hou, S.; Xie, T.; Zhang, Q.; Chen, X.; Wang, X.; Hu, L. Chartspiroton, a Tetracyclic Spiro-naphthoquinone Derivative from a Medicinal Plant Endophytic Streptomyces. Org. Lett. 2020, 22, 10, 3739-3743, https://doi.org/10.1021/acs.orglett.0c00696.

20. Jali, B. R.; Singh, M. W.; Baruah, J. B. Polymorphs of aromatic thiolato 1, 2 or 1,4-naphthoquinones. CrystEngComm, 2011, 13, 763-767, https://doi.org/10.1039/C0CE00591F. 
21. Jali, B. R.; Behura, R.; Barik, S. R.; Parveen, S.; Mohanty, S. P.; Das, R. A Brief Review: Biological Implications of Naphthoquinone Derivatives. Res J Pharm Technol. 2018, 11, 3698-3702, https://doi.org/10.5958/0974-360X.2018.00679.0.

22. Song, R.; Yu, B.; Friedrich, D.; Li, J.; Shen, H.; Krautscheid, H.; Huang, S. D.; Kim, M.-H. Naphthoquinonederivative as a synthetic compound to overcome the antibiotic resistance of methicillin-resistant $\mathrm{S}$. aureus. Commun Biol 2020, 3, 529, https://doi.org/10.1038/s42003-020-01261-0.

23. Jali, B. R.; Baruah, J. B. Recognition of a Bromide Ion by the Protonated Form of 2-(1H-Imidazol-2-ylthio)3-methylnaphthalene-1,4-dione. ChemPlusChem 2013, 78, 589-597, , https://doi.org/10.1002/cplu.201300042.

24. Peng, X.; Zhu, D.; Xiao, P. Naphthoquinone derivatives: Naturally derived molecules as blue-light-sensitive photoinitiators of photopolymerization. Eur. Polym. J. 2020, 127, 109569, https://doi.org/10.1016/j.eurpolymj.2020.109569.

25. Tong, L.; Goulet, M.-A.; Tabor, D. P.; Kerr, E. F.; Porcellinis, D. D.; Fell, E. M.; Aspuru-Guzik, A.; Gordon, R. G.; Aziz, M. J. Molecular Engineering of an Alkaline Naphthoquinone Flow Battery. ACS Energy Lett. 2019, 4, 1880-1887, https://doi.org/10.1021/acsenergylett.9b01321.

26. Sun, W. W.; Xu, Y.; Chen, X.; Xu, Y.; Wu, F.; Wang, Y. Reduced graphene oxide modified with naphthoquinone for effective immobilization of polysulfides in high-performance Li-S batteries. Chem. Eng. J. 2020, 383, 123111, https://doi.org/10.1016/j.cej.2019.123111.

27. Singh, W. M.; Jali, B. R.; Baruah, J. B. Iron (II) and Manganese (II) Complexes of 2-\{2-(3-Carboxypyridin2-yl) disulfanyl pyridine-3-carboxylic acid Through C-S Bond Cleavage. J Chem Crystallogr 2012, 42, 775782, https://doi.org/10.1007/s10870-012-0295-8.

28. Mohamady, S.; Gibriel, A. A.; Ahmed, M. S.; Hendy, M. S.; Naguib, B. H. Design and novel synthetic approach supported with molecular docking and biological evidence for naphthoquinonehydrazinotriazolothiadiazine analogs as potential anticancer inhibiting topoisomerase-IIB. Bioorg. Chem. 2020, 96, 103641, https://doi.org/10.1016/j.bioorg.2020.103641.

29. Paul, A.; Viswanathan, G. K.; Mahapatra, S.; Balboni, G.; Pacifico, S.; Gazit, E.; Segal, D. Antagonistic Activity of Naphthoquinone-Based Hybrids toward Amyloids Associated with Alzheimer's Disease and Type-2 Diabetes. ACS Chem. Neurosci. 2019, 10, 3510-3520, https://doi.org/10.1021/acschemneuro.9b00123.

30. Ravichandiran, P.; Boguszewska-Czubara, A.; Maslyk, M.; Bella, A. P.; Subramaniyan, S. A.; Johnson, P. M.; Shim, K. S.; Kim, H. G.; Yoo, D. J. Naphthoquinone-Based Colorimetric and Fluorometric Dual-Channel Chemosensor for the Detection of $\mathrm{Fe}^{2+}$ Ion and Its Application in Bio-Imaging of Live Cells and Zebrafish. ACS Sustainable Chem. Eng. 2019, 7, 17210-17219, https://doi.org/10.1021/acssuschemeng.9b03822.

31. Kosiha, A.; Munlo, K.; Parthiban, C.; Elango, K. P. Studies on the interaction of mononuclear metal(II) complexes of amino-naphthoquinone with bio-macromolecules. Mater. Sci. Eng. C. 2019, 94, 778-787, https://doi.org/10.1016/j.msec.2018.10.021.

32. Chadar, D.; Camilles, M.; Patil, R.; Khan, A.; Weyhermüller, T.; Salunke-Gawali, S. Synthesis and characterization of n-alkylamino derivatives of vitamin K3: Molecular structure of 2-propylamino-3-methyl1,4-naphthoquinone and antibacterial activities. J. Mol. Struct. 2015, 1086, 179-189, https://doi.org/10.1016/j.molstruc.2015.01.029.

33. Fosso, M. Y.; Chan, K. Y.; Gregory, R.; Chang, C.-W. T. Library synthesis and antibacterial investigation of cationic anthraquinone analogs. ACS Comb. Sci. 2012, 14, 231-235, https://doi.org/10.1021/co2002075.

34. Shrestha, J. P.; Subedi, Y. P.; Chen, L.; Chang, C.-W.T. A mode of action study of cationic anthraquinone analogs: a new class of highly potent anticancer agents. MedChemComm 2015, 6, 2012-2022, https://doi.org/10.1039/C5MD00314H.

35. Li, Y.; Jiang, J.-G. Health functions and structure-activity relationships of natural anthraquinones from plants. Food Funct. 2018, 9, 6063-6080, https://doi.org/10.1039/C8FO01569D.

36. Haruna, K.; Kanezaki, H.; Tanabe, K.; Dai, W. M.; Nishimoto, S. Effects of structural modification on the DNA binding properties and photo-induced cleavage reactivity of propargylic sulfones conjugated with an anthraquinone structure. Bioorg. Med. Chem. 2006, 14, 4427-4432, https://doi.org/10.1016/j.bmc.2006.02.036.

37. Rajendran, A.; Gandhidasan, R.; Murugesan, R. Photosensitisation and photoinduced DNA cleavage by four naturally occurring anthraquinones. J. Photochem. Photobiol., A. 2004, 168, 67-73, https://doi.org/10.1016/j.jphotochem.2004.02.014. 
38. Kemegne, G. A.; Mkounga, P.; Ngang, J. J. E.; Kamdem, S. L. S.; Nkengfack, A. E. Antimicrobial structure activity relationship of five anthraquinones of emodine type isolated from Vismia laurentii. BMC Microbiol. 2017, 17, 1-8, https://doi.org/10.1186/s12866-017-0954-1.

39. Lin, B. C.; Harris, D. R.; Kirkman, L. M. D.; Perez, A. M.; Qian, Y.; Schermerhorn, J. T.; Hong, M. Y.; Winston, D. S.; Yang, C. H.; Deng, L. F. Synthesis and biological evaluation of rhein amides as inhibitors of osteoclast differentiation and bone resorption. Eur. J. Med. Chem. 2016, 123, 769-776, https://doi.org/10.1016/j.ejmech.2016.08.004.

40. Mbatchou, V. C.; Tchouassi, D. P.; Dickson, R. A.; Annan, K.; Mensah, A. Y.; Amponsah, I. K.; Jacob, J. W.; Cheseto, X.; Habtemariam, S.; Torto, B. Mosquito larvicidal activity of Cassia tora seed extract and its key anthraquinones aurantio-obtusin and obtusin. Parasites Vectors 2017, 10, 1-8, https://doi.org/10.1186/s13071-017-2512-y.

41. Choudhari, D.; Lande, D. N.; Chakravarty, D.; Gejji, S. P.; Das, P.; Pardesi, K. R.; Satpute, S.; SalunkeGawali, S. Reactions of 2,3-dichloro-1,4-naphthoquinone with aminophenols: evidence for hydroxy benzophenoxazine intermediate and antibacterial activity. J. Mol. Struct 2019, 1176, 194-206, https://doi.org/10.1016/j.molstruc.2018.08.066.

42. Y1ldı, M. Design, synthesis, characterization, and antimicrobial activity of novel piperazine substituted 1,4benzoquinones. J. Mol. Struct 2020, 1203, 127422, https://doi.org/10.1016/j.molstruc.2019.127422.

43. Kumla, D.; Sousa, E.; Marengo, A.; Dethoup, T.; Pereira, J. A.; Gales, L.; Silva, J. F.; Costa, P. M.; Mistry, S.; Silva, A. M. S.; Kijjoa, A. 1,3-Dioxepine and spiropyran derivatives of viomellein and other dimeric naphthopyranones from cultures of Aspergillus elegans KUFA0015 and their antibacterial activity. Phytochemistry 2021, 181, 112575, https://doi.org/10.1016/j.phytochem.2020.112575.

44. Pereira, S. V. S. de; Oliveira, C. B. S. de.; Fumagalli, F.; Emery, F. da. S.; Silva, N. B. da.; Andrade-Neto, V. F. de. Cytotoxicity, hemolysis and in vivo acute toxicity of 2-hydroxy-3-anilino-1,4-naphthoquinone derivatives. Toxicol. Rep. 2016, 3, 756-762, https://doi.org/10.1016/j.toxrep.2016.09.007.

45. Saruul, E.; Murata, T.; Selenge, E.; Sasaki, K.; Yoshizaki, F.; Batkhuu, J. An antibacterial ortho-quinone diterpenoid and its derivatives from Caryopteris mongolica. Bioorg. Med. Chem. Lett. 2015, 25, 2555-2558, https://doi.org/10.1016/j.bmcl.2015.04.048.

46. Swapnaja, K. J. M.; Yennam, S.; Chavali, M.; Poornachandra, Y.; Kumar, C. G.; Muthusamy, K.; Jayaraman, V. B.; Arumugam, P.; Balasubramanian, S.; Sriram, K. K. Design, synthesis and biological evaluation of diaziridinyl quinone isoxazole hybrids. Eur. J. Med. Chem. 2016, 117, 85-98, https://doi.org/10.1016/j.ejmech.2016.03.042.

47. Ezeokonkwo, M. A.; Ugwuona, F. O.; Ugwu, I. C. Synthesis and Antibacterial Studies of Some Alkynylated Benzo[a]phenoxazin-5-one and 1,4-Naphthoquinone Derivatives. Asian J. Chem. 2015, 27, 3843-3850, https://doi.org/10.14233/ajchem.2015.19012.

48. Bayrak, N.; Yıldırım, H.; Tuyun, A. F.; Kara, E. M.; Celik, B. O.; Gupta, G. K.;. Synthesis, Biological, and Computational Study of Naphthoquinone Derivatives Containing Heteroatoms. J. Chem. Soc.Pak. 2016, 38, 1211-1221.

49. Kacmaz, A.; Acar, E. T.; Atun, G.; Kaya, K.; Sigirci, B. D.; Bagcigil, F. Synthesis, Electrochemistry, DFT Calculations, Antimicrobial Properties and X-ray Crystal Structures of Some NH- and/or S- Substituted-1,4quinones. Chemistry Select 2018, 3, 8615-8623. https://doi.org/10.1002/slct.201801155.

50. Rau, G.; Cretu, F. M.; Andrei, A. M.; Pisoschi, C. G.; Mogosanu, G. D.; Boroghina, A.; Banita, I. M.; Stanciulescu, C. E. Farmacia, 2015, 63, 665-669.

51. Novais, J. S.; Campos, V. R.; Silva, A. C. J. A.; de Souza, M. C. B. V.; Ferreira, V. F.; Keller, V. G. L.; Ferreira, M. O.; Dias, F. R. F.; Vitorino, M. I.; Sathler, P. C.; Santana, M.V.; Resende, J. A. L. C.; Castroa, H. C.; Cunha, A. C. Synthesis and antimicrobial evaluation of promising 7-arylamino-5,8-dioxo-5,8dihydroisoquinoline-4-carboxylates and their halogenated amino compounds for treating Gram-negative bacterial infections. RSC Adv., 2017, 7, 18311-18320, https://doi.org/10.1039/C7RA00825B.

52. Yıldırım, H.; Bayrak, N.; Tuyun, A. F.; Kara, E. M.; Çelik, B. O.; Gupta, G. K. 2,3-Disubstituted-1,4naphthoquinones containing an arylamine with trifluoromethyl group: synthesis, biological evaluation, and computational study. RSC Adv., 2017, 7, 25753-25764, https://doi.org/10.1039/C7RA00868F.

53. Ibis, C.; Ozsoy-Gunes, Z.; Tuyun, A. F.; Ayla, S. S.; Bahar, H.; Stasevych, M. V.; Musyanovych, R. Y.; Komarovska-Porokhnyavets, O; Novikov,V. Synthesis, antibacterial and antifungal evaluation of thio- or piperazinyl-substituted 1,4-naphthoquinone derivatives. J. Sulphur Chem. 2016, 37, 477-487, https://doi.org/10.1080/17415993.2016.1187734. 
54. Wang, D.; Zhang, W.; Wang, T.; Li, N.; Mu, H.; Zhang, J.; Duan, J. Unveiling the Mode of Action of Two Antibacterial Tanshinone Derivatives. Int. J. Mol. Sci. 2015, 16, 17668-17681, https://doi.org/10.3390/ijms160817668.

55. Rajasekar, S.; Krishna, T. P. A.; Tharmalingam, N.; Andivelu, I.; Mylonakis, E. Metal-Free C-H Thiomethylation of Quinones Using Iodine and DMSO and Study of Antibacterial Activity. Chemistry Select 2019, 4, 2281-2287, https://doi.org/10.1002/slct.201803816.

56. Novais, J. S.; Moreira, C. S.; Silva, A. C. J. A.; Loureiro, R. S.; Figueiredo, A. M. S.; Ferreira, V. F.; Castro, H. C.; da Rocha, D. R. Antibacterial naphthoquinone derivatives targeting resistant strain Gram-negative bacteria in biofilms. Microb. Pathog. 2018, 118, 105-114, https://doi.org/10.1016/j.micpath.2018.03.024.

57. Molee, W.; Phanumartwiwath, A.; Kesornpun, C.; Sureram, S.; Ngamrojanavanich, N.; Ingkaninan, K.; Mahidol, C.; Ruchirawat, S.; Kittakoop, P. Naphthalene Derivatives and Quinones from Ventilago denticulata and Their Nitric Oxide Radical Scavenging, Antioxidant, Cytotoxic, Antibacterial, and Phosphodiesterase Inhibitory Activities. Chem Biodivers 2018, 15, e1700537, https://doi.org/10.1002/cbdv.201700537. 